\title{
Hair Transplant for Eyebrow Restoration
}

\author{
Rajendrasingh J. Rajput ${ }^{1}$ \\ ${ }^{1}$ Hair Transplant Surgeon, Fellow ISHRS, IAT \\ Indian J Plast Surg 2021;54:489-494.
}

\begin{abstract}
Address for correspondence Dr. Rajendrasingh Rajput, MS, MCh, 1002, Satguru Sanskar, plot 19, TPS IV, 3rd Road, Off Turner Road, Bandra West, Mumbai 400050, Maharashtra, India (e-mail: drrajeshrajput@gmail.com).
\end{abstract}

\begin{abstract}
Keywords

- Eyebrow

- FUE

- Transplant

Eyebrow restoration can be best done with individual single hair follicles grafts harvested by FUE. Understanding the male and female eyebrow allows us to plan an aesthetic reconstruction, requiring 70 to 120 grafts. Selection of donor hair, angle of placement, alignment and direction within the rows influences the results, as does scarring and perfusion of the skin. Details of planning, technique, anesthesia and postoperative care, as well as drawbacks of older methods, are discussed in the article.
\end{abstract}

\section{Introduction}

Eyebrows contribute to complete the animation of the face. Three important muscles of facial expression are inserted into the dermis under the eyebrows, orbicularis oculi, corrugators and occipitofrontalis. ${ }^{1}$ Eyebrows also prevent sweat from trickling down onto the eyes. ${ }^{2}$ Okuda is known to have done eyebrow transplants in $1939 .^{3}$ Just thinning of eyebrows may not be an adequate indication for planning a transplant and risking a mismatch. It is worth mentioning that thinning eyebrows can often respond to low-dose oral minoxidil (LDOM; 2.5-5 mg per day), although this is an offlabel use. ${ }^{4}$

\section{Indications}

Common indications for surgical restoration of eyebrows are thermal burns, acid burns, trauma, alopecia areata, frontal fibrosing alopecia, trichotillomania, scarring alopecias, hypothyroidism, and Hanson's disease. ${ }^{5-7}$

\section{Case Selection}

Postburns or posttraumatic eyebrow restoration is planned only after all other functional deformities of the face are corrected, ${ }^{8}$ the scars are mature, and no more procedures are required for the face. Any further procedure for scar revision, flaps, skin grafts, and tissue expanders can shift the level of the reconstructed eyebrow, making them tilted or asymmetrical. During such time, the patient will have to manage with an eyebrow pencil. In alopecia areata, frontal fibrosing alopecia, scaring alopecias, and trichotillomania, a biopsy or an expert opinion and clinical conformation is awaited to make sure there are no active signs of the disease before planning the eyebrow restoration.

\section{Patient Counseling}

The patient should be counseled for realistic expectations, as there could be 5 to $10 \%$ loss of grafts and misdirection of grafts; also, frequent trimming of the grafts would be required every 2 to 3 weeks, patient may have to use vaseline to push the grafts along the direction of the brow and regulate the direction of growth, and there may be irregular or wavy growth due to scar tissue. Most of the times, a second sitting is requirement to add density, and partial use of eyebrow pencil may still be required to enhance the looks at regular intervals. There is possibility that the original disease may activate and destroy the transplanted eyebrows. A template of the eyebrows agreed preoperatively with the patient should be created on cloth or mackintosh to be autoclaved and used during the procedure. Photographs with the planned markings are part of the patient records. published online

December 14, 2021
DOI https://doi.org/ 10.1055/s-0041-1739253. ISSN 0970-0358.

\footnotetext{
(c) 2021. Association of Plastic Surgeons of India. All rights reserved. This is an open access article published by Thieme under the terms of the Creative Commons Attribution-NonDerivative-NonCommercial-License, permitting copying and reproduction so long as the original work is given appropriate credit. Contents may not be used for commercial purposes, or adapted, remixed, transformed or built upon. (https://creativecommons.org/ licenses/by-nc-nd/4.0/)

Thieme Medical and Scientific Publishers Pvt. Ltd., A-12, 2nd Floor, Sector 2, Noida-201301 UP, India
} 


\section{Characteristics of a Natural Eyebrow}

The eyebrows consist of serial arched rows (5-7 rows) of hair growing along the superior orbital margin. The eyebrows hair are short, thin but stiff, nonpliable with a slight curve along the body, and tapering toward the end. Caucasian eyebrows are thicker than scalp hair, while Asian eyebrow are thinner than scalp hair. ${ }^{3}$ Male eyebrows may be 5 to $6 \mathrm{~cm}$ long and 1 to $1.5 \mathrm{~cm}$ wide, whereas female eyebrows are $4.5-$ $5 \mathrm{~cm}$ long. ${ }^{9}$

The eyebrow is constituted of three parts, from medial to lateral, head, body and tail. ${ }^{9}$ The medial most or head constitutes approximately 10 to $15 \%$ or 0.6 to $0.8 \mathrm{~cm}$ of the eyebrow, is tilted below the orbital margin, and has one or two staggered rows of hair oriented vertically. The head has few short, light hair; most women and some men may not desire to reconstruct or highlight this part very much. Restoration of the head can be partial or even skipped in the final design. The middle part or the body constitutes approximately $60 \%$ or 2.5 to $3 \mathrm{~cm}$ of the brow, ${ }^{9}$ which gradually arches upward and lies on the orbital margin, with hair along the upper border pointing downward and outward. The body is the most dense, wide and crucial part of the eyebrow restoration. Crisscross placing along the upper and lower borders to overlap in the center, along the longitudinal axis of the eyebrow, is the best plan for a well-defined aesthetic eyebrow restoration. The lateral third or the tail constitutes approximately 20 to $25 \%$ (less than one forth) or 1.2 to $1.7 \mathrm{~cm}$ of the eyebrow. The tail lies above the orbital margin, is directed more horizontally and outward, and reaches beyond the lateral canthus. Eyebrow hair normally tends to be less dense laterally than medially; thus, loss of eyebrow hair from any cause is easily noticeable in the lateral portion. According to the layout and direction of the hair, there are two types of eyebrow patterns. ${ }^{10,11}$

\section{Two Eyebrow Patterns}

\section{Triple Pattern Brow}

Medial hair growing vertical, followed by gradually increasing outward and lateral inclination, being directed downward and outward in the middle third and finally downward and horizontal in the lateral third (-Fig. 1).

\section{Crisscross Brow}

Having hair in the upper half and lower half, growing crisscross toward each other. The body of the brow is the thickest area. The brows may be less dense medially and taper out laterally (-Fig. 2 ).

\section{Planning the Shape and Layout of the Eyebrow}

Pattern and shape of the opposite brow or old photograph can serve as a guide. An eyebrow slanting downward gives an elderly look, and eyebrow arched higher gives a surprised look. An eyebrow placed low medially and raised laterally gives an angry look. Lateral end should be at the same

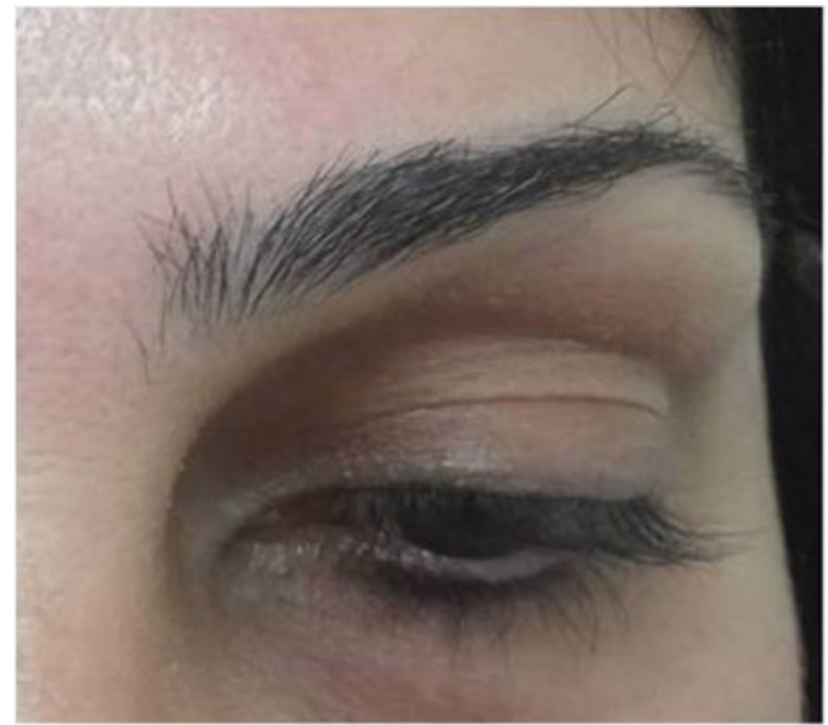

Fig. 1 Natural layout and eyebrow pattern.

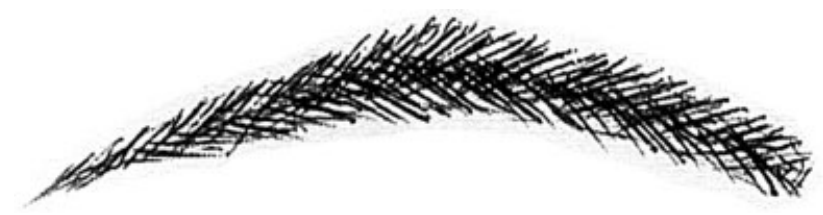

Fig. 2 Diagrammatic representation of crisscross brow.

horizontal level as the medial end or marginally higher than the medial end. A horizontal brow divides a long face and makes it look rounded or shorter, while a curved brow can make a round face look longer.

Female brows should be created thinner, as the patients are encouraged to thread, pluck and shape their opposite brow before coming for the surgery or draw the eyebrows they desire. Female eyebrow is slimmer and curved, usually require 2 to 3 rows of hair, with 70 to 90 grafts. Readymade templates are now available for microblading or eyebrow tattoo, which may be used for the planning. The female eyebrow begins slightly away from the medial canthus, is thinner and more "C-" shaped, attaining maximum curvature at the outer border of the limbus. The results of unilateral eyebrow restoration in a postburn scar are seen in -Fig. $\mathbf{3 a}$ and $\mathbf{3 b}$. Female patients should be made aware that the transplanted brow has thicker follicles, and these are difficult to reshape with threading or plucking. 6

Male eyebrow begins close to the glabella, slightly medial to the medial canthus, or along the level of the alar margin, maintaining an inter canthal distance equal to the width of the eye. The eyebrow begins medially almost below the orbital margin, then the upper border curves gradually upward above the orbital margin, while the lower margin is less curved and follows along the orbital margin. The body of the eyebrow is the thickest and widest part, consisting of 5 to 7 rows. The male eyebrow is less arched, reaches its highest curve at the lateral border of the limbus, and then 

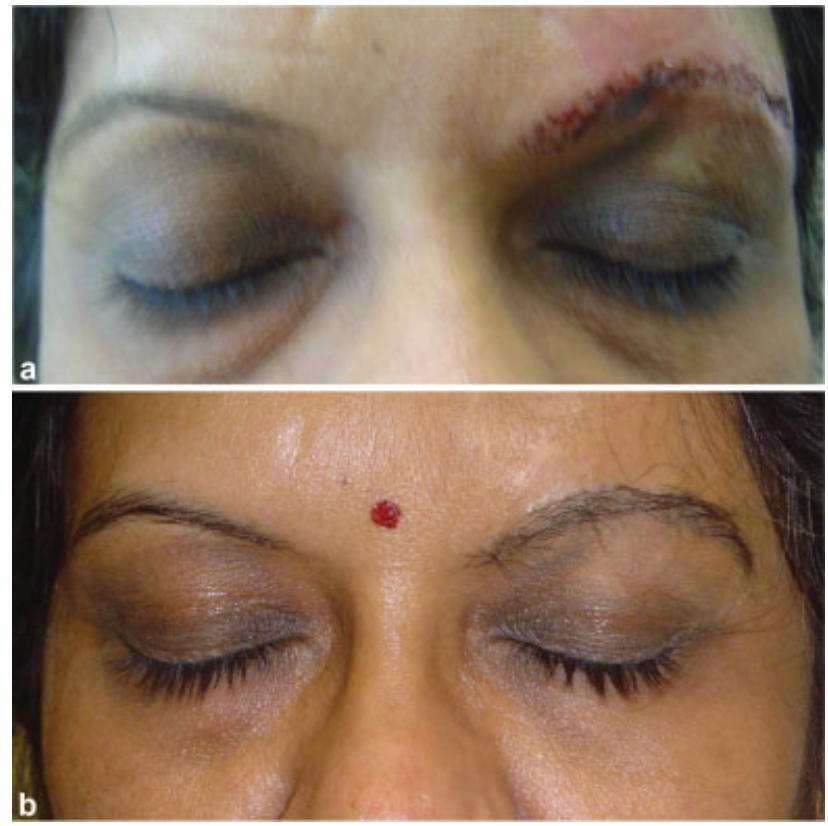

Fig. 3 (a) Posttraumatic scar left eyebrow pre. (b) Posttraumatic eyebrow restoration result.

drops down gradually above the lateral orbital rim, tapering into a tail and reaching beyond the lateral canthus. The medial and lateral ends should be at the same horizontal level or the tail may end marginally higher. ${ }^{9}$

\section{Anesthesia and Laboratory Tests}

Eyebrow area is innervated by infratrochlear, supratrochlear, supraorbital, lacrimal, and zygomaticotemporal nerves. Regional infiltration with local anesthetic solution is most effective. A lidocaine sensitivity test is performed to check for reactivity. We prefer to dilute the available $2 \%$ lidocaine to $1 \%$, add $1 \mathrm{~mL} \mathrm{1:1000}$ adrenaline, and add triamcinolone $10 \mathrm{mg}$ which prevents postoperative edema. Routine laboratory tests CBC, HIV, HbsAg, blood sugar, clinical stability of blood pressure, and cardiac status should be evaluated.

\section{Review of Options and Suitable Donor Sites}

Previous methods of eyebrow restoration are not recommended. Eyebrow reconstruction has been attempted with use of strip grafts and temporal artery-based flaps. These have resulted in too wide or too narrow flaps, loss of hair along the borders of the flap, leaving an unaesthetic, visible suture line, loss of hair on the ischemic and tips of the flaps, gradual fibrosis, loss of hair growth, scarring, change in the direction of hair growth, and unnatural appearance. ${ }^{12}$

Please do not be tempted to perform eyebrow restoration in alopecia totalis or universalis with artificial hair implants. ${ }^{13}$ These have always remained in trial and never delivered definitive results. Artificial hair has always led to one or more complications such as repeated infection, pustules, discharging sinuses, granulomas, scarring, ${ }^{13}$ no softness, no curve, and thick unnatural bristles jutting out of the skin like thorn pricks with pitting on the base. Body hairs have prolonged telogen, unreliable regrowth, and tendency to curl and distort. Body hair is not a choice of eyebrow restoration.

Follicular unit transplantation is now regarded as the best option for eyebrow restoration. ${ }^{14}$ Thin, slow growing hair single follicles are selected from the area below the occipital protuberance or the from the midparietal scalp. The author does not prefer the nape of the neck as donor, due to the possibility of reverse balding in men and considering that women may want to tie the hair in a bun or ponytail, exposing the hairline along the nape of the neck. Eyebrows require selective use of single hair dissected out of a scalp strip or extracted individually as single hair units and placed artistically as micrografts to create the eyebrows. A good eyebrow should recreate natural curve, characters, and pattern.

\section{FUE Technique of Harvesting Grafts for Eyebrow Restoration}

Follicular unit extraction (FUE) is the method of choice for selectively harvesting single hair grafts (-Fig. 4), with minimal donor morbidity, not easily noticeable scars, minimal pain, faster healing, and low downtime. In our experience, the best suitable punch is a $0.8-\mathrm{mm}$ sharp, manual or motorized punch. A horizontal strip of scalp hair below the occipital protuberance or along the midtemporal parietal area, measuring $1.5-2 \mathrm{~cm} \times 12-15 \mathrm{~cm}$, is trimmed, leaving the hair 3- to 5-mm long, in order to prepare for FUE harvesting. Leaving the hair 3 to $5 \mathrm{~mm}$ long helps in identification of the natural curve to align the grafts for a uniform natural aesthetic appeal. If adequate single hair follicles are not available, subfollicular harvesting ${ }^{9,10}$ or selective split harvesting of one hair out of the natural two to three hair units is practiced.

\section{FUT Technique of Harvesting Grafts for Eyebrow Restoration}

The other technique rarely used is follicular unit transplant (FUT). In FUT, a horizontal, elliptical strip of scalp $1 \mathrm{~cm} \times 4$ to $5 \mathrm{~cm}$, below the occipital protuberance, is excised and the wound closed in two layers, leaving a thin cosmetic scar. Such

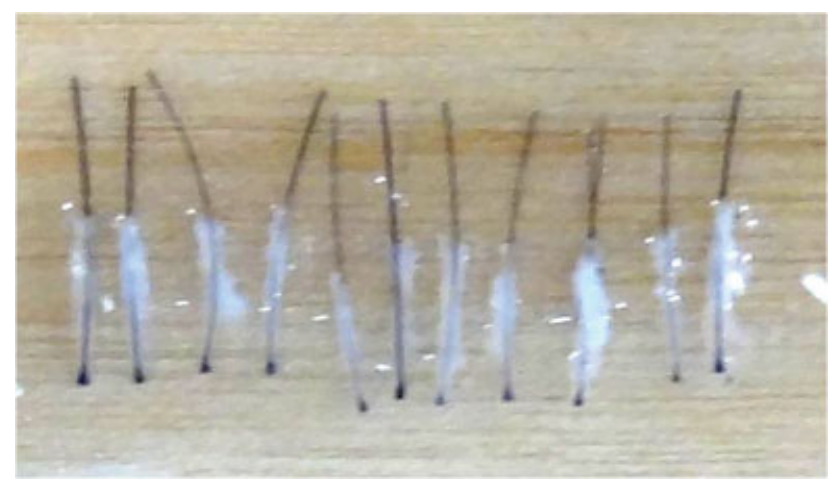

Fig. 4 Single hair grafts for eyebrow restoration. 
a strip can yield 400 grafts. Restricting the depth of the strip to the uppermost level of subdermal fat preserves lymphatics, veins and nerve endings, avoids neuromas and numbness, and ensures faster healing. The excised strip is dissected or slivered along the width under magnification, preferably under a stereomicroscopic microscope, creating single follicle rows of 8 to 10 follicles each called slivers. Natural follicular units having one, two, or three hairs are dissected out from the slivers and further divided into single hair grafts for eyebrow restoration. The grafts are trimmed of extra tissue and extra fat and deepithelized to promote healing.

\section{Holding Grafts Out of the Body}

Grafts harvested with a 0.8 FUE punch are slim and do not have much of extra tissue. No further trimming, fat removal, or deepithelization is required. All the grafts are inspected for damage and quality under a stereoscopic microscope. Any suspicious, transected, or damaged grafts are discarded, and more grafts are harvested to replace them. Grafts are counted and stored in chilled Ringer's lactate or saline at 4 to $8{ }^{\circ} \mathrm{C}$ to prevent dehydration. Grafts are grouped in 20 s in their order of harvesting, so that while implanting, we can follow the sequence of "first out first in" and reduce the out of body time. Thin grafts and thick coarse grafts are grouped differently. Thin grafts are preferred for a soft look along the upper border and along the tail of the eyebrow.

\section{Direction of the Grafts}

Premade slits are created flush to the skin to control the angle and direction of the single hair grafts. The author prefers coronal slits. At the medial end, a few staggered rows are created for the hair grafts to be placed pointing upward, and gradually the slits tilt outward and downward along the upper border in the body of the eyebrow, while becoming parallel to the axis of the eyebrow in the middle rows and then sloping downward in the lateral tail of the eyebrow. Two rows placed in this pattern along the upper border will define and highlight the curvature of the eyebrow. Then, two middle rows placed more horizontally, along the longitudinal axis of the eyebrow, give good body to the brow; finally, two rows of slits are created along the lower border to hold the grafts at right angles to the grafts from the upper border, creating a crisscross pattern and overlapping all along the central axis of the eyebrow. Each of the follicular unit survives like a microskin graft. About 150 to 200 single hair grafts are required for a thick, full male eyebrow restoration. Finer follicles are preferred for the upper border and the tail, while thicker coarse hair are placed in the midrow along the body of the eyebrow and along the lower border.

\section{Method of Placement of Grafts}

It is essential to place the grafts flush to the skin. Eyebrows placed at the slightest elevated angle grow out like canopies and look unacceptable. It is essential to create premade slits extremely flush along the skin. ${ }^{8-11,15}$ The premade slits can be created with special precut $1-\mathrm{mm}$ chisel blades or $19 \mathrm{G}$ and $20 \mathrm{G}$ hypodermic needles. ${ }^{10,11,15}$ Single right-angle bend on the needle or a "Z-" shaped bend can help in keeping the needle tracks flush to the skin. ${ }^{6-9}$ Then, you may implant the grafts into these slits in three ways. Use of implanter device, two forceps method to open or dilate the slit with one hand and place grafts with the other, and the third option being stick and place with hypodermic needle and forceps. With some practice, it is preferred to hold the grafts by the hair at the upper end and slide them into the premade slits. ${ }^{10,11,15}$ This is the "non root touch technique," ensuring minimum trauma to the delicate single hair grafts. The mobile, freely movable skin makes the slit making and graft placement difficult. An assistant should stretch and stabilize the skin for creating the premade slits during implantation for easy placement of the grafts. ${ }^{10,11,15}$ Today, the use of implanters offers good assistance for graft placement. The author prefers stick and place, using 20G and 19G hypodermic needles. The angle of the needle controls direction, depth, and layout of the hair. In scarring alopecias, the premade slit or the needle track must reach a vascular plane and show punctate bleeding through the premade slit track, so one can go deeper to reach a vascular plane. ${ }^{15}$ The punctate bleeding indicates adequate blood perfusion available to the graft and better survival. Natural skin elasticity holds the grafts in place. In the author's experience, thick scars with no elasticity can be cored out with a 0.8-mm, sharp FUE punch to create tracks that can easily accommodate the graft without pressure. Scar areas often require a second sitting. The follicle stem cells and pigment transferred into the scar often show improved skin quality and better results over time. ${ }^{10,11}$

\section{Spacing between the Grafts}

For eyebrow restoration, the micrografts are placed 1 to $1.5 \mathrm{~mm}$ apart $\mathrm{t}^{9,10,15}$ in staggered, interdigitating or brick wall overlapping pattern. In case of postburn and posttraumatic scars, it is best to wait for the scars to be mature, soft, and supple, making sure scars are not stuck to the underlaying bone and show blanching with quick refill on pressure. However, if you suspect reduced perfusion in the scar, the grafts have to be spaced at 2 to $4 \mathrm{~mm}$, in order to ensure good graft survival and then later add density in a second sitting. Hair transplant for eyebrow restoration can be planned over skin-grafted areas as well. In doubtful scars, the first sitting can be treated as a trial grafting.

\section{Postoperative Care}

Antibiotics and analgesics are advised for 2 days. No dressing is applied over the recipient areas, and these are left open. A thin layer of neosporin ointment or betadine ointment is applied to prevent drying on the grafts. Donor area is covered with betadine ointment and pad for 2 days. A saline spray every 3 hours keeps the grafts moist and prevents dehydration. Patients are instructed not to touch the grafts for the 
next 2 days. Cold compresses in the periorbital area are advised three times a day to prevent swelling. Patient is allowed a head bath and removal of the donor dressing on the third postoperative day. During bath, there should be no rubbing or friction over the grafts. A double-folded soft towel can be used to dab the areas or pat them dry. Gentle application of neosporin ointment or betadine with an earbud is advised twice a day from the third day. Patients are allowed or encouraged to touch and rub the grafts after 10 days to facilitate removal of the crusts. Some hair will be shed along with the crusts due to anagen effluvium. Complete regrowth of the grafts takes 6 to 8 months. Not all grafts regrow at the same time. Hair growth from 50 to $60 \%$ of the grafts commences at 3 to 4 months after the transplant. Another 20 to $25 \%$ grafts begin growth by 5 to 6 months and the last 10 to $15 \%$ grow at 7 to 8 months. Complete result is visible by 8 months. A second sitting should be planned only after 9 to 10 months.

\section{Complications}

Swelling, infection, bruising, folliculitis, and sebaceous cysts are extremely rare events in hair transplant for eyebrow reconstruction. ${ }^{8-11,15}$ Complications are mainly related to the curling of the hair, misdirection ( - Fig. 5), growth away from the skin like a canopy (-Fig. 6 ), poor density (-Fig. 7), poor growth and failure of growth of the grafts. Wavy, curled or distorted, misdirected, growth may occur from excessive handling, squeezing of grafts during implantation, poor circulation from scarred bed, and compression from the surrounding scar tissue. ${ }^{8-11,15}$ Aligning the hair down along the orbital margin, with vaseline or coconut oil, on the fingertips may help to regulate the direction. ${ }^{9,15}$ Sometimes, waiting for few months allows the hair to grow longer and often improves the direction of the transplanted hair. Patients are always counseled for a second sitting for density. The hair growth from grafts implanted in scarring alopecia and postburn and posttraumatic scar areas is often delayed. In our review of 63 cases of eyebrow restoration, ${ }^{15}$ and addition of a few more cases since the last report, ${ }^{15}$ it was concluded that $14 \%$ patients of eyebrow restoration could be corrected in one sitting, $77 \%$ required two sittings, and $9 \%$ required three sittings. Scalp hair grafts grow faster initially

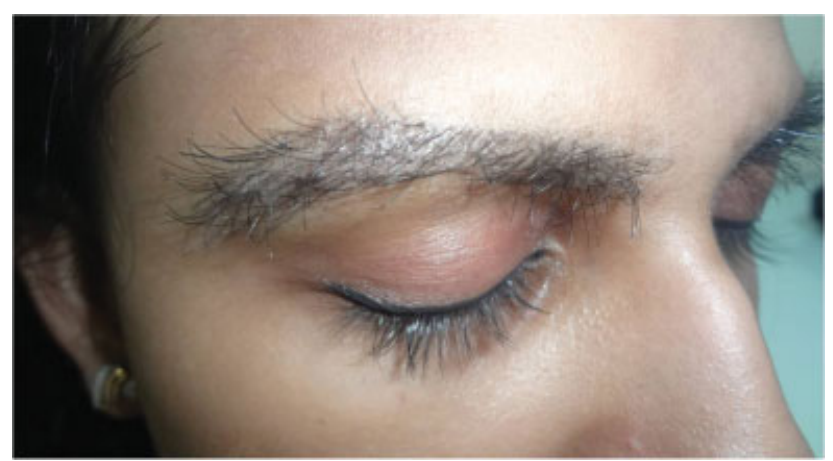

Fig. 5 Misdirection of the grafts.

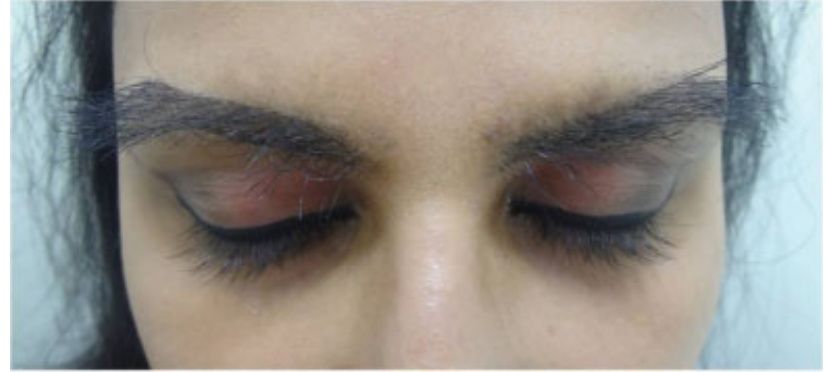

Fig. 6 Outward growth of grafts like a canopy.

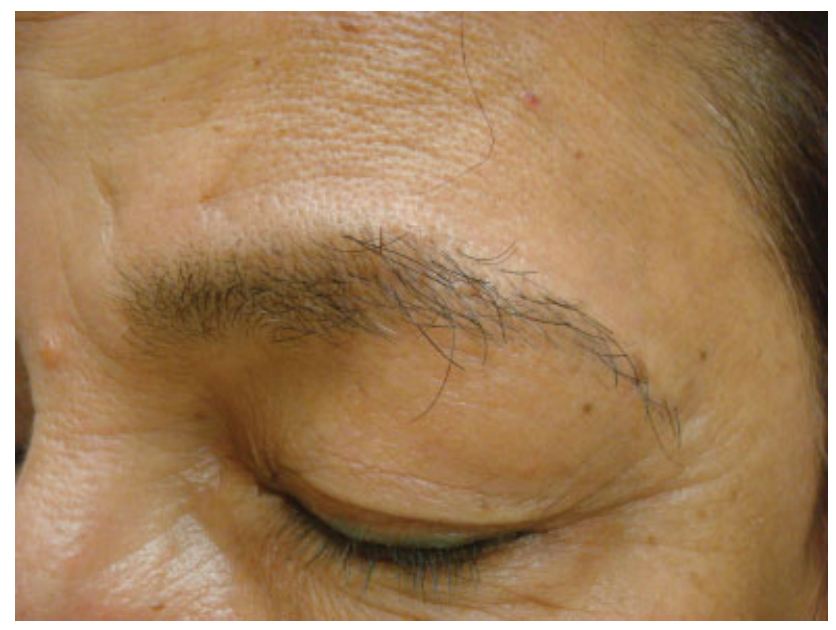

Fig. 7 Eyebrow poor density.

but are expected to gradually adapt to the recipient dominance and require trimming less often. ${ }^{9,15}$ Hair transplant for eyebrow restoration is time-consuming and laborious, but the results are rewarding.

\section{Conflict of Interest}

None declared.

\section{References}

1 Tong J, Lopez MJ, Patel BC. Anatomy, Head and Neck, Eye Orbicularis Oculi Muscle. Treasure Island (FL): StatPearls Publishing; 2021

2 Patel BC, Lopez MJ, Joos ZP. Anatomy, Head and Neck, Eyelash. Treasure Island (FL): StatPearls Publishing; 2021

3 Gupta J, Kumar A, Chouhan K, Ariganesh C, Nandal V. The science and art of eyebrow transplantation by follicular unit extraction. J Cutan Aesthet Surg 2017;10(02):66-71

4 Pirmez R, Spagnol Abraham L. Eyebrow regrowth in patients with frontal fibrosing alopecia treated with low-dose oral minoxidil. Skin Appendage Disord 2021;7(02):112-114

5 Van Loey NE, Van Son MJ. Psychopathology and psychological problems in patients with burn scars: epidemiology and management. Am J Clin Dermatol 2003;4(04):245-272

6 Gandelman MA. Technique for reconstruction of eyebrows and eyelashes. Semin Plast Surg 2005;19:153-158

7 Avram M. Follicular unit transplantation for male and female pattern hair loss and restoring eyebrows. Ophthalmol Clin North Am 2005;18(02):319-323, vii

8 Motamed S, Davami B. Eyebrow reconstruction following burn injury. Burns 2005;31(04):495-499 
9 Pathomvanich D. Anatomical restoration surgery of the eyebrow in Asian. Thai J Surg. 2004;25:47-52

10 Laorwong K, Pathomvanich D, Bunagan K. Eyebrow transplantation in Asians. Dermatol Surg 2009;35(03):496-503, discussion 503-504

11 Toscani M, Fioramonti P, Ciotti M, Scuderi N. Single follicular unit hair transplantation to restore eyebrows. Dermatol Surg 2011;37 (08):1153-1158

12 Mantero R, Rossi F. Reconstruction of hemi-eyebrow with a temporoparietal flap. Int Surg 1974;59(6-7):369-370
13 Mysore V. Controversy: synthetic hairs and their role in hair restoration? Int J Trichology 2010;2(01):42-44. Doi: 10.4103/0974-7753.66913

14 Gandelman M. Eyebrow and eyelash transplantation. In: Unger WP, ed. Hair Transplantation. New York: Marcel Deker; 1995

15 Rajput R. Eyebrow restoration. In: Mysore VA, ed. Hair Transplantation. Delhi, London, Panama, Philadelphia: Jaypee Brothers Medical Publishers (P) Ltd; 2016 